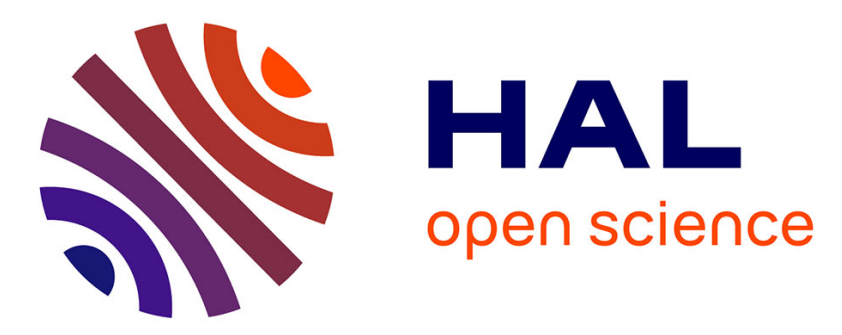

\title{
Continuously distributed control of plates by electric networks with PZT actuators
}

Stefano Vidoli, Francesco Dell'Isola

\section{To cite this version:}

Stefano Vidoli, Francesco Dell'Isola. Continuously distributed control of plates by electric networks with PZT actuators. 2010. hal-00514425

\section{HAL Id: hal-00514425 \\ https://hal.science/hal-00514425}

Preprint submitted on 2 Sep 2010

HAL is a multi-disciplinary open access archive for the deposit and dissemination of scientific research documents, whether they are published or not. The documents may come from teaching and research institutions in France or abroad, or from public or private research centers.
L'archive ouverte pluridisciplinaire HAL, est destinée au dépôt et à la diffusion de documents scientifiques de niveau recherche, publiés ou non, émanant des établissements d'enseignement et de recherche français ou étrangers, des laboratoires publics ou privés. 


\title{
Continuously distributed control of plates by electric networks with PZT actuators
}

\author{
Stefano Vidoli, Francesco dell'Isola \\ Dipartimento di Ingegneria Strutturale e Geotecnica, \\ Via Eudossiana 18, I-00184 Roma, Italia. \\ Email: vidoli@scilla.ing.uniroma1.it \\ isola@scilla.ing.uniroma1.it
}

\begin{abstract}
In this paper it is described a novel device aiming to control the mechanical vibrations of plates by means of a set of interconnected piezoelectric actuators. They are uniformly embedded in the plate and connect every node of an electric network to ground thus playing the two-fold role of capacitive element in the electric net and of couple suppliers. A mathematical model is introduced to describe the behavior of the system the time evolution of which is obtained as the solution of a system of partial differential equations. A self-resonance criterion is established which assures the possibility of electro-mechanical energy exchange. Finally the problem of vibration control in simply supported and clamped plates is addressed: the optimal net-impedance is determined. The results indicate that the proposed device can improve the performances of piezoelectric actuation.
\end{abstract}

\section{Introduction}

In [1], [2] the problem of controlling truss modular beams has been addressed: it is proven that the available piezoelectric actuators are in principle able to dampen their mechanical vibrations. In [3] the concept of continuously distributed control has been introduced and developed for one-dimensional beams. Such a control is obtained embedding in these structural members the actuators which are interconnected by an electric transmission line.

In the present paper it is proposed to control the vibrations in plates distributing in them a set of actuators continuously interconnected. However, being the plate a two-dimensional structural member, such an interconnection must be obtained by the new concept of electrical two-dimensional continuously distributed network. The mathematical difficulty (in comparison with [3]) to be confronted concerns the need of changing the kinematical descriptors of the electric state of the system. This difficulty goes along with the need of conceiving a way of suitably interconnecting the actuators which is intrinsically two-dimensional. Both these difficulties are circumvented introducing the field of e-state (the material time derivative of which is the electric potential) and the conception of a truly two-dimensional interconnection scheme among actuators the evolution of which parallels those valid for the plate. 


\subsection{Advantages of proposed continuous net-control sys- tems}

The common features of the already conceived control devices are represented by the differentiation between the sensing and the actuation systems and the localization of PZT actuators in few sites of the vibrating structure. Both are limits to the control efficiency; indeed, the first one implies the need of a coordinating active system, controlling the actuators actions in response to the inputs from the sensors, while the latter implies an optimal localization problem -for both actuators and sensors- the solutions of which depends on the particular mechanical vibration mode to be considered (see [8]). Moreover it is difficult to optimize the characteristics of the control system to obtain low equivalent impedances -these are required to allow for a relevant energy transformation from the mechanical to the electrical form- and efficiently drive the PZT actuators (see [6], [9]).

Some efforts to overcome the first of these drawbacks have been made. In particular, the concept of self-sensing actuator has been introduced (see [5]): an ad hoc electric circuit is connected to the piezoelectric patch allowing its twofold behavior. However every patch remains isolated and its electro-mechanical action has to be coordinated with the rest of the structure. Also, when a great number of actuators is used to control the shape of plates (see [7]), it was never conceived any kind of their interconnection via a circuital network.

Exploiting the concept of "parallelism" between mechanical structures and electric control systems, it is proposed to control a plate-like structure by means of a distribution of actuators connected to an electric transmission net: an internal resonance phenomenon, between structural modes and electric modes, is induced to obtain the maximum control efficiency.

The net-control system has two practical advantages: it requires lower performances to the PZT actuators and allows for a strong control action and shorter times to transfer the energy between electrical and mechanical forms. Moreover the net-control system bypasses the problems of the optimal positioning (of actuators and sensors) being able to manage all mechanical modes thought the same distributed configuration of its collocated actuators.

\subsection{Coarse description of the system}

We propose to control the plate with a distribution of piezoelectric actuators interconnected by means of electric impedances as shown in Figure 1: gray boxes represent the actuators while the black ones represent the electric impedances.

A possible electric connection scheme between actuators and the impedances is reproduced in Figure 2. We remark that the fundamental topological difference between the considered two-dimensional net and a transmission line: here a node is grounded by means of an actuation device and is connected to at least four other nodes. This circumstance mathematically is accounted for introducing a space partial differential equations governing the evolution of the electric state descriptors. 
From an electrical viewpoint the actuators can be considered as capacitances: one plate is connected to the net while the other is grounded. On the other hand, from a mechanical viewpoint, the actuators provide mechanical actions in response to electric inputs and contribute to the structural stiffness.

\section{Mathematical model}

To describe the main behavior of the proposed electro-mechanical system, a continuum model is introduced; this choice allows for a accurate description of vibrations only when the involved wave-lengths are not too small compared with the dimensions of the single actuator. We obtain a set of partial differential equations for the fields describing the electric and mechanical states starting from a postulated balance of power.

\subsection{Equation of motion}

We deal with a plate body $\mathcal{B}$ occupying the region $\mathcal{C}=\mathcal{S} \times \mathcal{I}$, where $\mathcal{S}$ is a plane surface and $\mathcal{I}$ the real interval $[-h, h]$. As usual the thickness $2 h$ is supposed to be small compared with the diameter of $\mathcal{S}$. The following balance of power

$$
\begin{array}{r}
\int_{\mathcal{S}} \int_{\mathcal{I}}(\mathbf{b} \cdot \dot{\mathbf{u}})+\int_{\partial \mathcal{S}} \int_{\mathcal{I}}(\mathbf{f} \cdot \dot{\mathbf{u}})+\int_{\mathcal{S}}(i \dot{\psi})+\int_{\partial \mathcal{S}}(\chi \dot{\psi})= \\
\int_{\mathcal{S}} \int_{\mathcal{I}}[\mathbf{S} \cdot(\operatorname{sym} \text { Grad } \dot{\mathbf{u}})]+\int_{\mathcal{S}}(\mathbf{I} \cdot \operatorname{grad} \dot{\psi}),
\end{array}
$$

must hold for every test field $(\dot{\mathbf{u}}, \dot{\psi})$. Here u represents the displacement field while $\dot{\psi}$ is the electric potential field: as a consequence $\dot{\mathbf{u}}$ is the velocity field and $\psi$ is the time-integral of the electric potential between the nodes and the ground. Moreover $\mathrm{b}$ and $\mathrm{f}$ are the body and surface external forces, $i$ and $\chi$ the body and surface current densities from the ground, $\mathbf{S}$ the stress tensor and $\mathbf{I}$ the current vector in the net, Grad and grad mean the gradient operators in $\mathcal{C}$ and $\mathcal{S}$ respectively.

According to the geometry of the body, the position vector is decomposed as:

$$
\mathbf{x}=\mathbf{r}+\zeta \mathbf{e}
$$

where $\mathbf{r}$ is position vector in $\mathcal{S}, \zeta \in \mathcal{I}$ and $\mathbf{e}$ is the unit vector perpendicular to $\mathcal{S}$. To deduce from the 3-dimensional Cauchy model of $\mathcal{B}$ the behavior of a bending plate we use the Kirchhoff-Love compatible identification procedure based on the following kinematical reduction map for displacements:

$$
\mathbf{u}(\mathbf{r}, \zeta)=w(\mathbf{r}) \mathbf{e}-\zeta \operatorname{grad} w(\mathbf{r})
$$


The function $w$ models the transverse displacement of the points of the plate. It is assumed that the electric potential drop $\dot{\psi}$ depends on $\mathbf{r}$ only. It follows that the infinitesimal deformation field is expressed as:

$$
\mathbf{E}=\operatorname{sym}(\operatorname{Grad} \mathbf{u})=-\zeta \operatorname{sym}(\operatorname{grad} \operatorname{grad} w) .
$$

Substituting in the eqn. (1) the reduction map (3) we obtain:

$$
\begin{array}{r}
\int_{\mathcal{S}}(b \dot{w}+\mathbf{B} \cdot \operatorname{grad} \dot{w}+i \dot{\psi})+\int_{\partial \mathcal{S}}(T \dot{w}+\mathbf{m} \cdot \operatorname{grad} \dot{w}+\chi \dot{\psi})= \\
\int_{\mathcal{S}}[\mathbf{M} \cdot \operatorname{sym}(\operatorname{grad} \operatorname{grad} \dot{w})+\mathbf{I} \cdot \operatorname{grad} \dot{\psi}]
\end{array}
$$

where

$$
\begin{aligned}
\mathbf{M} & =-\left.\int_{\mathcal{I}} \zeta \mathbf{S}\right|_{\mathcal{S}}, \quad \mathbf{B}=-\left.\int_{\mathcal{I}} \zeta \mathbf{b}\right|_{\mathcal{S}}, \quad b=\int_{\mathcal{I}} \mathbf{b} \cdot \mathbf{e}, \\
T & =\int_{\mathcal{I}}(\mathbf{f} \cdot \mathbf{e}), \quad \mathbf{m}=\int_{\mathcal{I}}-\left.\zeta \mathbf{f}\right|_{\mathcal{S}}
\end{aligned}
$$

are the dynamical actions in the reduced plate model. Applying the divergence theorem, we get:

$$
\begin{aligned}
& \int_{\mathcal{S}}[(b-\operatorname{div} \mathbf{B}-\operatorname{div} \operatorname{div} \mathbf{M}) \dot{w}+(i+\operatorname{div} \mathbf{I}) \dot{\psi}]+ \\
& \int_{\partial \mathcal{S}}\{[T+(\mathbf{B}-\operatorname{div} \mathbf{M}) \cdot \nu+] \dot{w}+(\mathbf{m}-\mathbf{M} \nu) \cdot \operatorname{grad} \dot{w}+(\chi-\mathbf{I} \cdot \nu) \dot{\psi}\}=0
\end{aligned}
$$

that must hold for every admissible velocity field $(\dot{w}, \dot{\psi})$; this condition leads to the following balance equations:

$$
\operatorname{div} \operatorname{div} \mathbf{M}+\operatorname{div} \mathbf{B}-b=0, \quad \operatorname{div} \mathbf{I}+i=0, \quad \text { on } \mathcal{S},
$$

and boundary conditions:

$$
\int_{\partial \mathcal{S}}\{[T+(\mathbf{B}-\operatorname{div} \mathbf{M}) \cdot \nu+] \dot{w}+(\mathbf{m}-\mathbf{M} \nu) \cdot \operatorname{grad} \dot{w}+(\chi-\mathbf{I} \cdot \nu) \dot{\psi}\}=0
$$

for the reduced model of the electro-mechanical plate.

Assuming that the body $\mathcal{B}$ is linear isotropic and homogeneous (so that we do not need to distinguish in the power balance the actual from the reference configuration) and that the network is linear and dissipative, we get

$$
\mathbf{S}=2 \mu_{L} \mathbf{E}+\lambda_{L}(\operatorname{tr} \mathbf{E}) \mathbf{1}, \quad \mathbf{b}=-\rho \ddot{\mathbf{u}}, \quad-\operatorname{grad} \dot{\psi}=L_{N} \dot{\mathbf{I}}+R_{N} \mathbf{I},
$$


where $\mu_{L}$ and $\lambda_{L}$ are the Lamè moduli, $\rho$ the mass density, $L_{N}$ and $R_{N}$ are respectively the net-inductance and net-resistance and 1 means the identity operator in $\mathcal{C}$. As a consequence the part of reduced constitutive equations which does not depend on piezoelectro-mechanical coupling (i.e. $\mathbf{M}_{m}$ and $i_{e}$ ) read as follows:

$$
\mathbf{M}_{m}=J_{\mathcal{I}}\left[2 \mu_{L} \operatorname{sym}(\operatorname{grad} \operatorname{grad} w)+\lambda_{L}(\operatorname{lapl} w) \hat{\mathbf{1}}\right], \quad i_{e}=\frac{k_{C}}{d^{2}} \ddot{\psi}
$$

where $J_{\mathcal{I}}=2 h^{3} / 3$, lapl means the laplacian operator in $\mathcal{S}, \hat{\mathbf{1}}$ means the identity operator in $\mathcal{S}$ and $k_{C}$ is the purely electric grounded capacitance, while $d^{2}$ represents the area of influence of the actuator (namely the area of the plate divided by the number of actuators). For the inertial terms from (11) and (6) we get

$$
\mathbf{B}=-J_{\mathcal{I}} \rho \operatorname{grad} \ddot{w}, \quad b=-2 h \rho \ddot{w} .
$$

However the piezoelectric actuators have a two-fold behavior: from a mechanical viewpoint they enhance the bending stiffness of the plate and produce bending moments in response to applied voltage; from an electric viewpoint they enhance the grounded capacitance per unit area of the electric net and produce a charge in response to applied curvatures.

Let us introduce a orthonormal coordinate system $\left(o, \mathbf{e}_{1}, \mathbf{e}_{2}\right)$ in $\mathcal{S}$. Concerning the part of bending moment tensor induced by the piezoelectric effect $\mathbf{M}_{e}$ we assume that its component expending power on the component $w_{, 12}$ of the curvature vanishes so that the following representation holds:

$$
\mathbf{M}_{e}=M_{11}\left(\mathbf{e}_{1} \otimes \mathbf{e}_{1}\right)+M_{22}\left(\mathbf{e}_{2} \otimes \mathbf{e}_{2}\right) .
$$

In addition we specify the properties of the single PZT actuator used by

$$
\left\{\begin{array}{c}
M_{11} \\
M_{22} \\
Q / d^{2}
\end{array}\right\}=\left[\begin{array}{ccc}
g_{m m} & 0 & -g_{m e} \\
0 & g_{m m} & -g_{m e} \\
g_{m e} & g_{m e} & g_{e e}
\end{array}\right]\left\{\begin{array}{c}
w_{, 11} \\
w_{, 22} \\
\dot{\psi}
\end{array}\right\}
$$

where $M_{i i}$ and $w_{, i i}$ are the bending moments and curvatures in the principal directions while $Q / d^{2}$ and $\dot{\psi}$ are the charge per unit area and voltage between the actuator plates. The constitutive eqn. (15) establishes that the actuators can exert electrically induced moments only in two orthogonal directions and that they can not exert "mixed" moments: the orthogonal system introduced accounts for this directionality. Moreover we have assumed that the piezoelectric stiffnesses in $\mathbf{e}_{1}$ and $\mathbf{e}_{2}$ are equal; this assumption seems reasonable when using PZT actuators exploiting Poisson effect.

Therefore the overall constitutive relations for $\mathbf{M}$ and $i$ read as follows:

$$
\begin{aligned}
\mathbf{M} & =\mathbf{M}_{m}+g_{m m}\left[w_{, 11}\left(\mathbf{e}_{1} \otimes \mathbf{e}_{1}\right)+w_{, 22}\left(\mathbf{e}_{2} \otimes \mathbf{e}_{2}\right)\right]-g_{m e} \dot{\psi} \hat{\mathbf{1}}, \\
i & =i_{e}+\frac{\dot{Q}}{d^{2}}=i_{e}+g_{e e} \ddot{\psi}+g_{m e} \text { lapl } \dot{w},
\end{aligned}
$$


Let us now introduce the bending stiffness and capacitance per unit area of the plate

$$
D_{P}=J_{\mathcal{I}}\left(2 \mu_{L}+\lambda_{L}\right), \quad C_{N}:=g_{e e}+\frac{k_{C}}{d^{2}}=: \frac{\left(k_{e e}+k_{C}\right)}{d^{2}} ;
$$

the balance equations in terms of kinematical fields becomes:

$$
\left\{\begin{array}{l}
D_{P} \text { dlapl } w+g_{m m}\left(w_{, 1111}+w_{, 2222}\right)-J_{\mathcal{I}} \rho \text { lapl } \ddot{w}+2 h \rho \ddot{w}-g_{m e} \text { lapl } \dot{\psi}=0 \\
-\operatorname{lapl} \psi+L_{N} C_{N} \ddot{\psi}+R_{N} C_{N} \dot{\psi}+R_{N} g_{m e} \text { lapl } w+L_{N} g_{m e} \text { lapl } \dot{w}=0
\end{array}\right.
$$

where lapl and dlapl are the laplacian and double laplacian operators in $\mathcal{S}$.

In order to find the dimensionless form of (19) we introduce as spatial characteristic length the diameter of the plate $\ell$, and define $v:=w / \ell, \phi:=\psi / \bar{V}$, the characteristic pulsation ${ }^{1} \omega=\frac{\pi}{\ell} \sqrt{\frac{D_{P}}{M_{P}}}$-being $M_{P}=2 \rho \ell^{2} h$ the total mass of the plate- so that:

$$
\left\{\begin{array}{l}
\frac{D_{P}}{2 h \rho \ell^{4} \omega^{2}} \Delta \Delta v+\frac{g_{m m}}{2 h \rho \ell^{4} \omega^{2}}\left(v_{, 1111}+v_{, 2222}\right)-\frac{h^{2}}{3 \ell^{2}} \Delta \ddot{v}+\ddot{v}-\frac{g_{m e} \bar{V}}{M_{P} \ell \omega} \Delta \dot{\phi}=0, \\
-\frac{1}{L_{N} C_{N} \ell^{2} \omega^{2}} \Delta \phi+\ddot{\phi}+\frac{R_{N}}{L_{N} \omega} \dot{\phi}+\frac{g_{m e}}{C_{N} \ell \omega \bar{V}} \Delta \dot{v}+\frac{R_{N} g_{m e}}{L_{N} C_{N} \bar{V} \ell \omega^{2}} \Delta v=0 .
\end{array}\right.
$$

Now $\triangle$ means the dimensionless laplacian operator, ()$_{, i}$ and the dot mean the dimensionless space and time derivative. In the application we will consider, we have

$$
\frac{g_{m m}}{2 h \rho \ell^{4} \omega^{2}} \ll \frac{D_{P}}{2 h \rho \ell^{4} \omega^{2}}, \quad \frac{h^{2}}{3 \ell^{2}}\|\triangle \ddot{v}\|_{H^{2}} \ll\|\ddot{v}\|_{H^{2}} ;
$$

the last inequality holds when the wave-length is much bigger than the plate thickness and when one considers only the lower spatial eigenmodes.

The characteristic e-state parameter $\bar{V}:=\sqrt{M_{P} / C_{N}}$ is chosen to maintain the symmetry, so that (20) becomes:

$$
\left\{\begin{array}{l}
\alpha \Delta \triangle v+\ddot{v}-\gamma \triangle \dot{\phi}=0 \\
-\beta \triangle \phi+\ddot{\phi}+\gamma \Delta \dot{v}+\delta \dot{\phi}+\delta \gamma \triangle v=0 .
\end{array}\right.
$$

where

$$
\begin{aligned}
& \alpha:=\frac{D_{P}}{M_{P} \ell^{2} \omega^{2}}=\frac{1}{\pi^{2}}, \quad \beta:=\frac{1}{L_{N} C_{N} \ell^{2} \omega^{2}}, \\
& \gamma:=\frac{g_{m e}}{\ell \omega} \sqrt{\frac{1}{M_{P} C_{N}}}, \quad \delta:=\frac{R_{N}}{L_{N} \omega}
\end{aligned}
$$

\footnotetext{
${ }^{1}$ This choice implies that the first mechanical mode in the case of a simply supported plate has a pulsation equal to $2 \pi$.
} 
are dimensionless numbers. Remark that when the electro-mechanical coupling parameter $\gamma$ vanishes, (22) reduces to the uncoupled system of the KirchhoffLove plate and membrane-like electric network equations

$$
\left\{\begin{array}{l}
\alpha \Delta \triangle v+\ddot{v}=0 \\
\ddot{\phi}+\delta \dot{\phi}=\beta \Delta \phi
\end{array}\right.
$$

\subsection{Partitioned modal analysis}

In this section we adapt to the set of equations (22) the reasonings developed in [3].

Let $\mathcal{H}_{m}$ and $\mathcal{H}_{e}$ be the subspaces of $\mathrm{L}^{2}(\mathcal{S})$, the space of $\mathcal{R}$-valued squareintegrable functions defined on $\mathcal{S}$ verifying suitable homogeneous boundary and smoothness conditions; let $v, \dot{v}, \ddot{v} \in \mathcal{H}_{m}$ and $\phi, \dot{\phi}, \ddot{\phi} \in \mathcal{H}_{e}$. Let $\mathbf{L}_{m m}$ and $\mathbf{L}_{e e}$ be linear self-adjoint differential operators on $\mathcal{H}_{m}$ and $\mathcal{H}_{e}$ respectively, and $\mathbf{G}_{m e}^{A}$ indicate the adjoint of the linear differential operator $\mathbf{G}_{m e}$ from $\mathcal{H}_{e}$ to $\mathcal{H}_{m}$. We consider the following evolutionary problem:

$$
\left\{\begin{array}{l}
\alpha \mathbf{L}_{m m}(v)+\ddot{v}-\gamma \mathbf{G}_{m e}(\dot{\phi})=0 \\
\beta \mathbf{L}_{e e}(\phi)+\ddot{\phi}+\gamma \mathbf{G}_{m e}^{A}(\dot{v})+\delta \dot{\phi}+\delta \gamma \mathbf{G}_{m e}^{A}(v)=0
\end{array}\right.
$$

starting from initial condition for $v$ and $\phi$. Remark that eqns. (22) has the structure of (26). The subscripts $m$ and $e$ stand for mechanical and electrical respectively.

In order to study the interaction between the electrical and mechanical components of state descriptors we introduce in $\mathcal{H}_{m}$ and $\mathcal{H}_{e}$ the eigenbases supplied by the spectral representations theorem for the self-adjoint operators $\mathbf{L}_{m m}$ and $\mathbf{L}_{e e}$ respectively. Therefore for every $v \in \mathcal{H}_{m}, \phi \in \mathcal{H}_{e}$ we have:

$$
\begin{array}{rlrl}
v & =\sum_{h} v_{h} m_{h}, \quad \phi=\sum_{k} \phi_{k} e_{k} \\
\mathbf{L}_{m m}(v) & =\sum_{h} \lambda_{h} v_{h} m_{h}, \quad & \mathrm{~L}_{e e}(\phi)=\sum_{k} \nu_{k} \phi_{k} e_{k} .
\end{array}
$$

Here $\lambda_{h}$ and $\nu_{k}$ respectively denote the eigenvalues of $\mathbf{L}_{m m}$ and $\mathbf{L}_{e e}, m_{h}$ and $e_{k}$ are the corresponding eigenfunctions, $v_{h}:=\left\langle v, m_{h}\right\rangle_{L^{2}}$ and $\phi_{k}:=\left\langle\phi, e_{k}\right\rangle_{L^{2}}$ are the time-dependent Fourier coefficients. If we define the scalars:

$$
\begin{aligned}
& C_{h k}:=\left\langle m_{h}, \mathbf{G}_{m e}\left(e_{k}\right)\right\rangle_{L^{2}}, \\
& C_{k h}:=\left\langle e_{k}, \mathbf{G}_{m e}^{A}\left(m_{h}\right)\right\rangle_{L^{2}}=\left\langle\mathbf{G}_{m e}\left(e_{k}\right), m_{h}\right\rangle_{L^{2}}=C_{h k},
\end{aligned}
$$

and consider that $m_{h}$ and $e_{k}$ are bases of $\mathcal{H}_{m}$ and $\mathcal{H}_{\boldsymbol{e}}$ as eigenfunctions of self-adjoint operators, eqns. (26) can be written:

$$
\left\{\begin{array}{l}
\ddot{v}_{h}+\alpha \lambda_{h} v_{h}-\gamma \sum_{k} C_{h k} \dot{\phi}_{k}=0 \\
\ddot{\phi}_{h}+\beta \nu_{h} \phi_{h}+\delta \dot{\phi}_{h}+\gamma \sum_{k} C_{k h}\left(\delta v_{k}+\dot{v}_{k}\right)=0,
\end{array} \quad h, k=1,2,3 \ldots\right.
$$


Equations (31) clearly show that the influence on the mode $m_{h}$ exerted by the mode $e_{k}$ is measured by the matrix $C_{h k}$ that we can regard as a modal $e \rightarrow m$ coupling matrix. In an absolutely similar way $C_{k h}$ represents the modal $m \rightarrow e$ coupling matrix. It is now easy to formulate the following

\section{Criterion for electro-mechanical coupling}

A necessary condition for the presence of electro-mechanical energy exchange between $e_{k}$ and $m_{h}$ modes is

$$
C_{h k}=\left\langle m_{h}, \mathbf{G}_{m e}\left(e_{k}\right)\right\rangle_{L^{2}} \neq 0 .
$$

\section{Results}

\subsection{Analytical solution for the simply supported square plate}

Consider a simply supported square plate of side $\ell$, connected to an electric net grounded on the boundary; in this case the boundary conditions for eqns. (22) become

$$
v=0, \quad \mathbf{M} \nu=0, \quad \phi=0,
$$

on each side of the square domain $\mathcal{S}$. The eigenvalues of the purely mechanical and electrical operators

$$
\mathbf{L}_{m m}(f)=\triangle \triangle f, \quad \mathbf{L}_{e e}(g)=-\triangle g
$$

are respectively given by

$$
\lambda_{k}=\pi^{4}\left(i_{k}^{2}+j_{k}^{2}\right)^{2}, \quad \nu_{k}=\pi^{2}\left(i_{k}^{2}+j_{k}^{2}\right),
$$

while the corresponding eigenfunctions are

$$
e_{k}=\sin \left(i_{k} \pi x_{1}\right) \sin \left(j_{k} \pi x_{2}\right), \quad m_{h}=\sin \left(i_{h} \pi x_{1}\right) \sin \left(j_{h} \pi x_{2}\right),
$$

being $x_{i}=\left(\mathbf{r} \cdot \mathbf{e}_{i}\right) / \ell$. Here $i_{-}$and $j_{-}$relate the two different mode labeling according to Table 1.

\begin{tabular}{|l|r|l|l|l|l|l|l|l|l|}
\cline { 2 - 10 } \multicolumn{1}{c|}{} & $k=1$ & 2 & 3 & 4 & 5 & 6 & 7 & 8 & 9 \\
\hline$i_{k}$ & 1 & 1 & 2 & 2 & 1 & 3 & 2 & 3 & 3 \\
\hline$j_{k}$ & 1 & 2 & 1 & 2 & 3 & 1 & 3 & 2 & 3 \\
\hline
\end{tabular}

Table 1: Modes labeling

Recalling that in this case $\mathbf{G}_{m e}(g)=\Delta g$, we can compute the matrix $C_{h k}$ getting:

$$
C_{h k}=\int_{\mathcal{S}} \triangle\left(e_{k}\right) m_{h}=-\pi^{2}\left(i_{k}^{2}+j_{k}^{2}\right) \int_{\mathcal{S}} e_{k} e_{h}=-\pi^{2}\left(i_{k}^{2}+j_{k}^{2}\right) \delta_{h k}
$$


Note that, because the eigenfunctions are mutually orthogonal, the coupling matrix is diagonal; thus, the coupling exists only between corresponding modes!

The system (22) is decomposed into an uncoupled sequence of $1-1$ problems like the following:

$$
\left\{\begin{array}{l}
\ddot{v}_{h}+\alpha \lambda_{h} v_{h}-\gamma C_{h h} \dot{\phi}_{h}=0, \\
\ddot{\phi}_{h}+\beta \nu_{h} \phi_{h}+\delta \dot{\phi}_{h}+\gamma C_{h h}\left(\delta v_{h}+\dot{v}_{h}\right)=0,
\end{array} \quad h=1,2,3 \ldots\right.
$$

or omitting to write the subscript $h$ :

$$
\left\{\begin{array}{l}
\ddot{v}+A v-C \dot{\phi}=0 \\
\ddot{\phi}+B \phi+C \dot{v}+D \dot{\phi}+C D v=0
\end{array}\right.
$$

where $A=\alpha \lambda_{h}, B=\beta \nu_{h}, C=\gamma C_{h h}$ and $D=\delta$.

Therefore in the case of simply supported rectangular plate the membranelike electric network is able to couple one mechanical mode exactly with one electrical mode so that one can get a self-resonance tuning the electric net parameters. However the parameters appearing in (38) depend on the considered mode number: in general the aforementioned tuning will cause self-resonance of only few pairs of electro-mechanical modes.

\subsubsection{Non damped energy exchange}

In order to establish the conditions assuring the maximal energy exchange between the mechanical and electrical states, one could develop the general treatment delineated in the case of one-dimensional electro-mechanical structures in [3]. However for seek of simplicity we consider here a simplified version of that treatment, studying the 1-1 coupling through eqns. (39) and then extending the results to multiple couplings.

First of all we analyze the non-dissipative case $(D=0)$ of eqns. (39). Its solution, starting from a purely mechanical initial data $v_{0}$, is the following modulated signal:

$$
v(t)=V_{1} \cos \left(\alpha_{1} t\right)+V_{2} \cos \left(\alpha_{2} t\right), \quad \phi(t)=\Phi_{1} \sin \left(\alpha_{1} t\right)+\Phi_{2} \sin \left(\alpha_{2} t\right),
$$


where:

$$
\begin{aligned}
& \alpha_{1}=\sqrt{\frac{1}{2}\left[\left(C^{2}+A+B\right)-\sqrt{\left(C^{2}+A+B\right)^{2}-4 A B}\right]} \\
& \alpha_{2}=\sqrt{\frac{1}{2}\left[\left(C^{2}+A+B\right)+\sqrt{\left(C^{2}+A+B\right)^{2}-4 A B}\right]} \\
& V_{1}=\frac{u_{0}}{2}\left(1+\frac{C^{2}-A+B}{\sqrt{\left(C^{2}+A+B\right)^{2}-4 A B}}\right) \\
& V_{2}=\frac{u_{0}}{2}\left(1-\frac{C^{2}-A+B}{\sqrt{\left(C^{2}+A+B\right)^{2}-4 A B}}\right) \\
& \Phi_{1}=\frac{\alpha_{1}^{2}-A}{\alpha_{1} C} V_{1}, \quad \Phi_{2}=\frac{\alpha_{2}^{2}-A}{\alpha_{2} C} V_{2} .
\end{aligned}
$$

In the hypothesis $C^{2} \ll A$, with simple manipulations one can find the low frequency analogical components of the modulated signal i.e.

$$
I_{\mathrm{MAX}}=\left(V_{1}+V_{2}\right) \cos \left(\frac{\alpha_{1}-\alpha_{2}}{2} t\right), I_{\min }=\left(V_{1}-V_{2}\right) \sin \left(\frac{\alpha_{1}-\alpha_{2}}{2} t\right),
$$

respectively representing the envelops of the maxima and minima, and, as usual, related to the energy contents.

Since we are interested to the most efficient exchange of energy between the mechanical and electrical forms, we seek for the values of the parameter $B$ that minimize the amplitude of $I_{\min }$ and the time $T_{\mathrm{tr}}$ elapsed to transform the maximal possible amount of initial energy in electrical form

$$
\min _{B}\left|I_{\min }\right|=\min _{B}\left|V_{1}-V_{2}\right|, \quad \min _{B} T_{\mathrm{tr}}=\max _{B}\left|\alpha_{1}-\alpha_{2}\right|
$$

These conditions imply respectively

$$
B_{1}=A-C^{2}, \quad B_{2}=A+C^{2},
$$

indicating that in the interval $\left(B_{1}, B_{2}\right)$ we get self-resonance. Everywhere in the following we will assume that the self-resonance condition is $B=A$.

Let us now consider the ratio

$$
\mathbb{k}=\frac{C^{2}}{A}=\frac{g_{m e}^{2}}{D_{P} C_{N}}
$$

which is much smaller than 1 in the applications considered here. This number plays an important rôle in determining the pulsation of the low-frequency analogical components in the chosen self-resonance condition. Indeed the dimensionless time interval $\left.T_{\mathrm{tr}}\right|_{B=A}$ needed to transform the mechanical energy of the considered mode into electrical is given as a function of $\mathbf{k}$ (see Figure 4) by:

Fig. 3

Fig. 4 


$$
\left.T_{\mathrm{tr}}\right|_{B=A}=\frac{1}{2(\sqrt{1+\sqrt{\mathrm{k}}}-\sqrt{1-\sqrt{\mathbb{k}}})} .
$$

Recall that $T_{\mathrm{tr}}$ is a dimensionless time and $T_{\mathrm{tr}}=1$ represents one period of the first purely mechanical mode.

The self-resonance condition $B=A$ also implies:

$$
\beta \simeq \frac{\alpha \lambda_{h}}{\nu_{h}}=i_{h}^{2}+j_{h}^{2} .
$$

Note that $\beta$ depends on the net-inductance $L_{N}$ that is a tunable parameter. Thus we can simply tune the net-inductance value to couple two modes which, verifying the criterion (32), can be made resonant; the optimal value $L_{N h}^{*}$ to couple the $h$-th modes is

$$
L_{N h}^{*}=\frac{1}{\left(i_{h}^{2}+j_{h}^{2}\right) C_{N} \ell^{2} \omega^{2}}=\frac{1}{\left(i_{h}^{2}+j_{h}^{2}\right)\left(k_{e e}+k_{C}\right) N_{A} \omega^{2}} ;
$$

here $N_{A}=\ell^{2} / d^{2}$ is the total number of actuators.

The energy flux related to the solution (40) for $B=A$, is visualized in Figure 5 where the thick gray line represents the electric energy.

Note that in Figure 5 there are four different kind of energies involved:

Fig. 5

1. the mechanical elastic energy, $A v^{2} / 2$;

2. the mechanical kinetic energy, $\dot{v}^{2} / 2$;

3 . the electric inductive energy, $B \phi^{2} / 2$;

4. the electric capacitive energy, $\dot{\phi}^{2} / 2$;

and that only the total sum of these energies is constant.

\subsubsection{Damped energy exchange}

The characteristic polynomial of eqns. (39) is now

$$
P(s):=s^{2} C^{2}+s D C^{2}+\left(s^{2}+A\right)\left(s^{2}+s D+B\right)=0,
$$

its complex roots representing the damping ratios (real parts) and the pulsations (imaginary parts) of the associated eigenfunctions.

In Figure 6 a pair of roots (the other pair is the complex conjugate) are drawn as functions of the ratio $D / C$; the gray scale measures the electromechanical coupling of the associated eigenvectors: black means comparable electro-mechanical contents. Moreover, the projections of the curves on the planes $\{D / C,-\operatorname{Re}\}$ and $\{D / C, \operatorname{Im}\}$ are drawn.

We observe that:

1. Increasing the ratio $D / C$ (i.e. the net-resistance) definitively leads to the uncoupling of the electro-mechanical wave-forms. 
2. The projections on the plane $\{D / C,-\mathrm{Re}\}$ show a maximum of the damping ratio of the root relative to wave-form turning to purely mechanical content when $D / C$ tends to infinity. This circumstance allows for the determination of the critical value for the parameter $D$ proportional to the net-resistance.

3. The projections on the plane $\{D / C, \operatorname{Im}\}$ show that varying $D$ the eigenfrequencies attain a minimal distance; when $D / C$ tends to infinity the mechanical eigenfrequency tends to $\sqrt{A}$, the electric one vanishes.

A further description of the locus of the roots of the characteristic polynomial (49) can be obtained introducing the following one

$$
Q(s):=s^{2} C^{2}+\left(s^{2}+A\right)\left(s^{2}+s D+A\right) .
$$

As $P$ and $Q$ are real polynomial their roots can be paired by conjugation having coincident (negative because $D$ is positive) real parts. Let us call these real parts $\delta_{P m}, \delta_{P e}$ and $\delta_{Q m}, \delta_{Q e}$. It can be proven that

$$
A>0, \quad B>0, \quad D>0, \quad C>0 \quad \Longrightarrow \quad\left(\delta_{Q m}, \delta_{Q e}\right) \subset\left(\delta_{P m}, \delta_{P e}\right) .
$$

Therefore an upper bound for the maximum mechanical damping ratio is obtained by the real parts of the roots of $Q$ when $D=2 C$ and its dimensionless value is $1 / 2$. These considerations are summarized in Figure 7 where the bold lines represents the real parts of the root of $Q$ and the dashed ones represent the real parts of the roots of $P$ when $B \rightarrow A$.

Using the approximated condition $D=2 C$ obtained by means of $Q$ and recalling the definitions of $D, C, C_{h h}$, and $L_{N}^{*}$ we get the following estimated values for the optimal net-resistance:

$$
R_{N}^{*}=\frac{2 \pi^{2} g_{m e}}{C_{N} \ell^{3} \omega^{2}} \sqrt{\frac{1}{M_{P} C_{N}}}=\frac{2 \pi^{2} g_{m e}}{\left(k_{e e}+k_{C}\right) N_{A}^{3 / 2} \omega^{2}} \sqrt{\frac{1}{M_{P}\left(k_{e e}+k_{C}\right)}} .
$$

Note that $R_{N}^{*}$ is, in this case, independent of the mode number: this fact will not hold true when a clamped plate will be considered. Remark that both the values of the optimal inductance $L_{N h}^{*}$ and resistance $R_{N}^{*}$ decrease when a "more distributed" net-control system is considered (namely when the number of actuators $N_{A}$ increases).

When a dissipative net is considered -in eqn. (39) $D \neq 0$, the energy, once transformed in electric form, is now dissipated and only a fraction transforms back in mechanical form.

In comparing Figures 5 and 8 one should consider that the displayed energy dissipation is obtained for a value of the net-resistance which is not the optimal one. 


\subsection{Numerical solution for the clamped plate}

When considering a completely clamped plate a technical difficulty arises in applying the criterion for electro-mechanical coupling. Indeed there is not a treatable closed form for the eigenfunctions of $\mathbf{L}_{m m}$; however one can use their close approximation represented by the product of the eigenfunctions of a clampedclamped beam (see the first row in Figure 9); on the other side we still consider an membrane-like net electrically grounded on its boundary so that the eigenfunctions of $\mathbf{L}_{e e}$ will not change (second row in Figure 9). The labeling of the basis in $\mathbf{L}^{2}(\mathcal{S})$ we have just chosen trivially parallels the previous one (see Table 1).

We will apply the coupling criterion to this set of approximated eigenfunctions. The coupling matrix $C$ is no more diagonal, however, due to the similarities between the modes, the matrix $C$ is quasi-diagonal. Figure 10 shows a representation of the matrix $C$ by means of the gray scale (blank cell means a vanishing value); the first nine mechanical and the first nine electrical modes are considered.

The novelty in the case of clamped plate is represented by the possibility of coupling one mechanical modes with different electrical ones (if the corresponding $C_{h k}$ is non vanishing): indeed note that a simple tuning of the net-inductance allow to make coincident any two frequencies; however all the electric frequencies are shifted together (see Figure 11).

In order to use the analysis of the previous section in the present case, we list in Table 2

- the eigenvalues $\nu_{k}$ of the spatial operator $\mathbf{L}_{e e}(g)=\Delta g$ with $g=0$ on $\partial \mathcal{S}$,

- the eigenvalues $\lambda_{k}$ of the spatial operator $\mathbf{L}_{m m}(v)=\Delta \Delta v$ simply supported on $\partial \mathcal{S}$,

- the eigenvalues $\lambda_{k}^{(c)}$ of the spatial operator $\mathbf{L}_{m m}(v)=\Delta \Delta v$ clamped on $\partial \mathcal{S}$

- the ratios $c_{k}=\lambda_{k}^{(c)} / \lambda_{k}$.

$\begin{array}{lrrrrrrrrr} & k=1 & 2 & 3 & 4 & 5 & 6 & 7 & 8 & 9 \\ \nu_{k}=\left(i_{k}^{2}+j_{k}^{2}\right) & 2 & 5 & 5 & 8 & 10 & 10 & 13 & 13 & 18 \\ \lambda_{k}=\left(i_{k}^{2}+j_{k}^{2}\right)^{2} & 4 & 25 & 25 & 64 & 100 & 100 & 169 & 169 & 324 \\ \lambda_{k}^{(c)} & 13.4 & 55.8 & 55.8 & 121.6 & 180.2 & 180.2 & 282.6 & 282.6 & 501.0 \\ c_{k} & 3.35 & 2.23 & 2.23 & 1.90 & 1.80 & 1.80 & 1.67 & 1.67 & 1.55\end{array}$

Table 2: Compared eigenvalues 
The eigenvalues $\lambda_{k}^{(c)}$ have been obtained estimating the Rayleight ratio on the approximated eigenfunctions. Table 2 is used to determine the optimal inductance and resistance values, namely

$$
L_{N h}^{*}=\frac{M_{P}}{c_{h}\left(i_{h}^{2}+j_{h}^{2}\right) C_{N} \pi^{2} D_{P}}, \quad R_{N h}^{*}=\frac{2 g_{m e}}{c_{h} C_{N} \ell D_{P}} \sqrt{\frac{M_{P}}{C_{N}}} .
$$

We conclude this section with two plots of the frequency-response functions. In them the net-inductances and resistances are tuned respectively on the optimal values $\left(L_{N 1}^{*}, R_{N 1}^{*}\right)$ and $\left(L_{N 2}^{*}, R_{N 2}^{*}\right)$. The black and gray lines plot the norms of the purely mechanical and purely electric part of the response function, while the dashed one represents the norm of the coupling part of the response function.

The numerical results confirm the validity of the coupling criterion and as a consequence of the optimal impedance values. Indeed the electro-mechanical coupling shows maximal peaks with wider frequency bandwidths when these optimal values are chosen.

In the numerical simulations the values in Table 3 were used. The assumed performances of PZT actuators are realistic: they concern the ACX actuators QP20W.

\begin{tabular}{|l|l|l|l|}
\hline$\ell=1 \mathrm{~m}$ & $h=1 \mathrm{~mm}$ & $N_{A}=7 \times 7=49$ & $g_{m e}=28.10^{-5} \mathrm{~N} \mathrm{~V}^{-1}$ \\
\hline$\rho=2700 \mathrm{Kg} \mathrm{m}^{-3}$ & $E_{Y}=7010^{9} \mathrm{GPa}$ & $k_{e e}=0.6 \mu \mathrm{F}$ & $k_{C}=1 \mu \mathrm{F}$ \\
\hline
\end{tabular}

Table 3: Mechanical and electrical parameters.

\section{Conclusions}

The device which is proposed in this paper is based on the concept of global synergic response of a set of PZT actuators to a given mechanical modal form. This global response is obtained by conceiving an electric network interconnecting the single actuators. The single electric signal produced by one of them is the potential drop between a node of the electric net and the ground. Thus PZT actuation is accompanied by an electric waveform which evolves together with the mechanical one. In order to study the performances of the conceived system a mathematical model of its dynamic behavior has been developed: it is obtained by means of an homogenization procedure and therefore it gives only rough predictions when short wavelengths of the electro-mechanical signals are considered. In the framework of this model it is proven a criterion assuring electro-mechanical coupling: it allows for the determination of the netimpedances maximizing the electromechanical energy exchange. The efficiency of the device is indicated by the very low damping ratios which it shows when the optimal net impedance is chosen. This is its main advantage when compared with the devices based on the concept of concentrated actuation (see $[10]$ and [5]). Also a remarkable decrease of the needed impedance for getting electro-mechanical coupling is obtained. 


\section{References}

[1] dell'Isola F., Vidoli S.: Continuum modelling of piezoelectro-mechanical truss beams: an application to vibration damping, Archive of Applied Mechanics 68, 1-19, 1998.

[2] dell'Isola F., Vidoli S.: Bending-waves damping in truss beams by electrical transmission line with PZT actuators, Archive of Applied Mechanics 68, 626-636, 1998.

[3] Vidoli S., dell'Isola F.: Modal coupling in one-dimensional electromechanical structured continua, Acta Mechanica, 141, 1-2, 2000.

[4] Reed M., Simon B.: Methods of modern mathematical physics, Boston: Academic Press 1980.

[5] Fuller C.R., Elliott S.J., Nelson P.A.: Active control of vibration, London: Academic Press 1996.

[6] Hagood N.W., Von Flotov A.: Damping of structural vibrations with piezoelectric materials and passiv electrical networks, J. of Sound and Vibration, 146 (2), 243-268, 1991.

[7] Batra R.C., Ghosh K.: Deflection control during dynamic deformations of a rectangular plate using piezoceramic elements, AIAA Journal 33 (8), pp. 1547-1549, 1995.

[8] Formica N.C., Balis Crema L., Galeazzi C., Morganti F.: Vibration control of satellite panels by means of piezoelectric elements, in Dynamics and Control of Structures in Space III, Eds. Kirk C.L. and Inman D.J., London: Computational Mechanics Publications, 1996.

[9] Bondoux D.: Piezo-damping of light structures: modelling and experimental results, in Dynamics and Control of Structures in Space III, Eds. Kirk C.L. and Inman D.J., London: Computational Mechanics Publications, 1996.

[10] Guran A., Inman D.J.: Wave motion, intelligent structures and nonlinear mechanics, Singapore: World Scientific 1995. 


\section{Figure Captions}

1. Assembled plate and network.

2. Electric connection scheme.

3. Low-frequency analogical components.

4. Elapsed time to transfer energy.

5. Energy exchange.

6. Characteristics roots.

7. Plot of the polynomial $Q(s)$.

8. Energy dissipation.

9. Functional basis for the clamped plate.

10. Representation of the coupling matrix.

11. Electric and mechanical frequencies.

12. Frequency-response function (optimal impedance for mode 1).

13. Frequency-response function (optimal impedance for modes 2 and 3). 


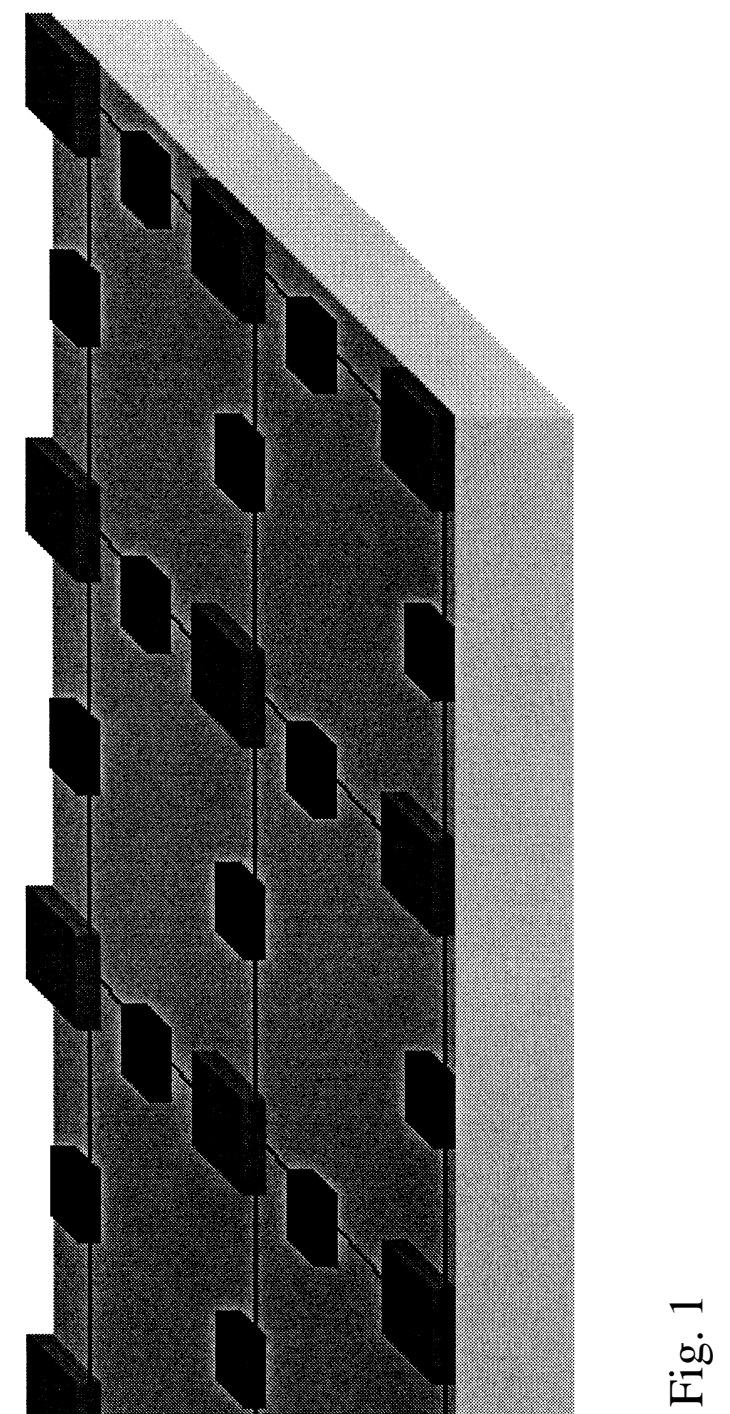




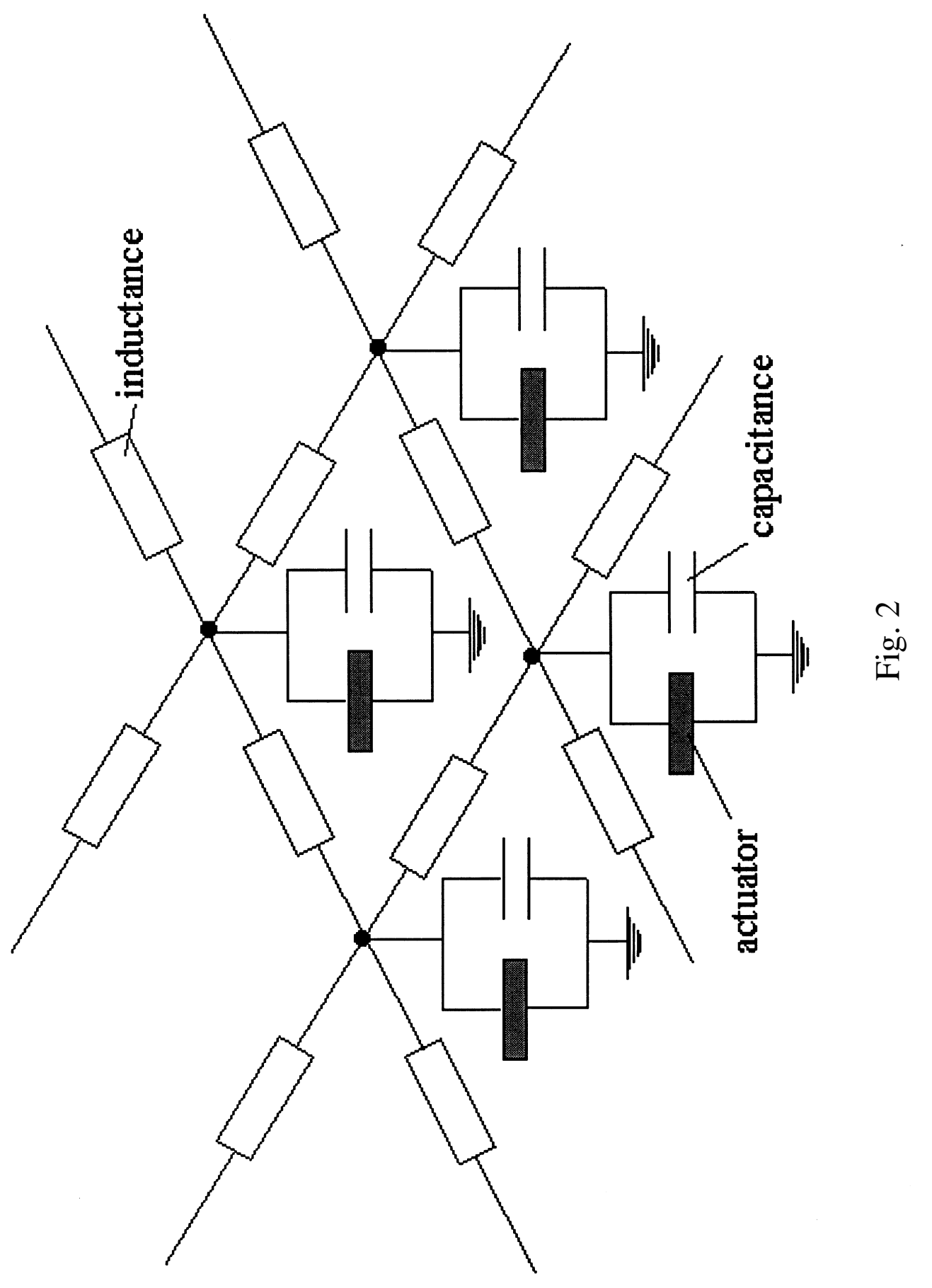




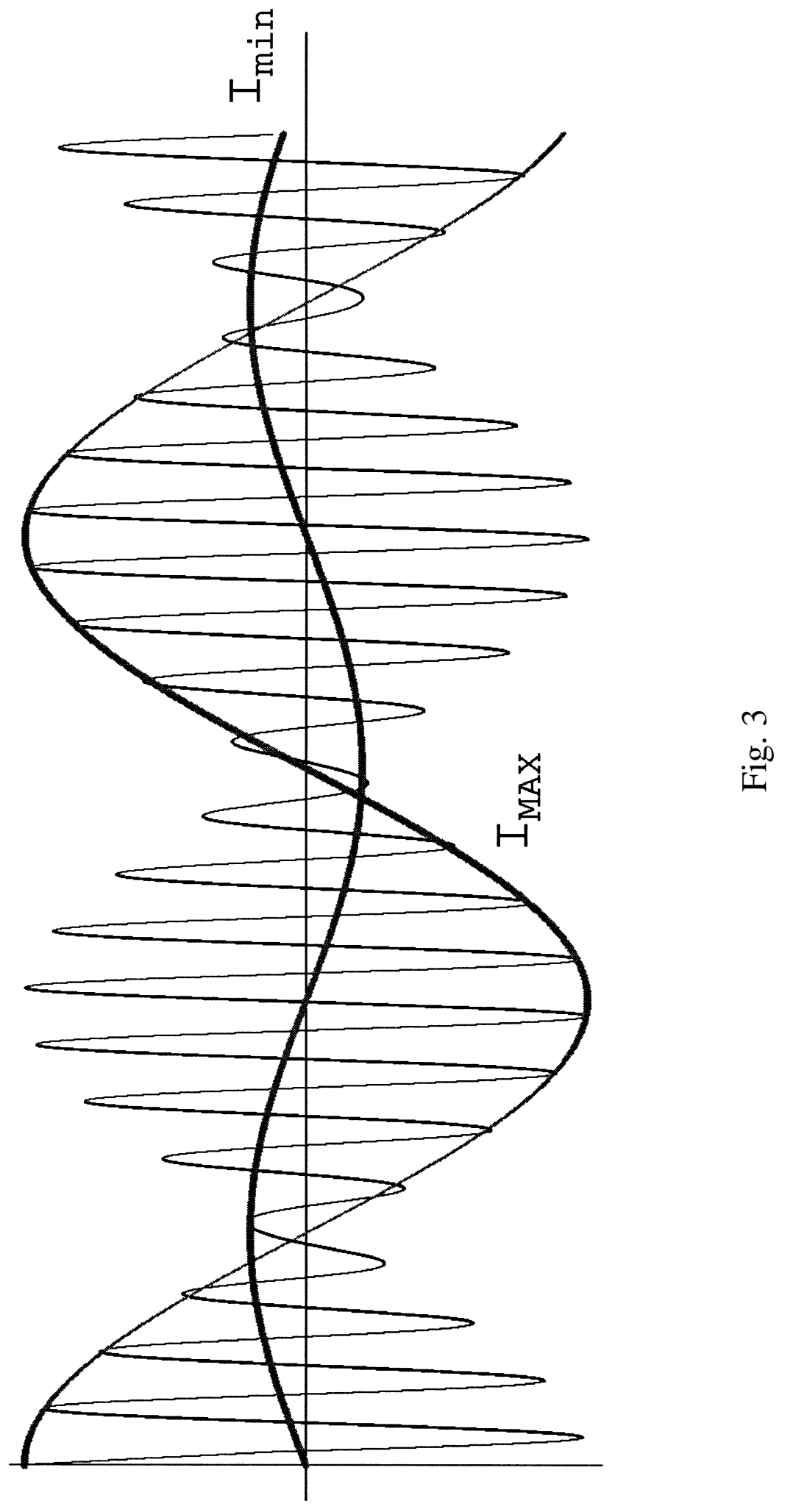



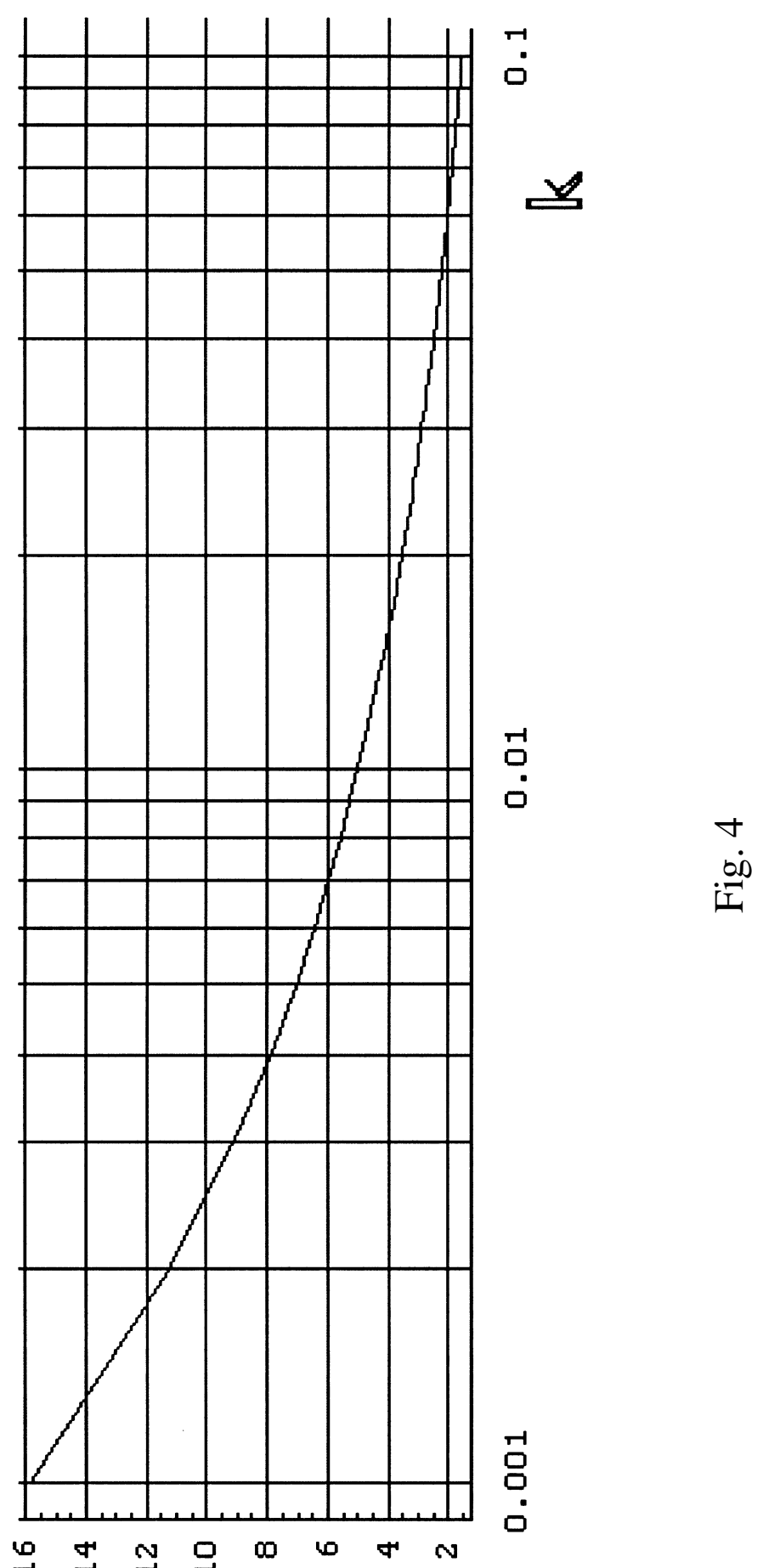

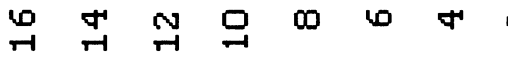

$\frac{\|_{0}^{\pi}}{E^{\infty}}$ 


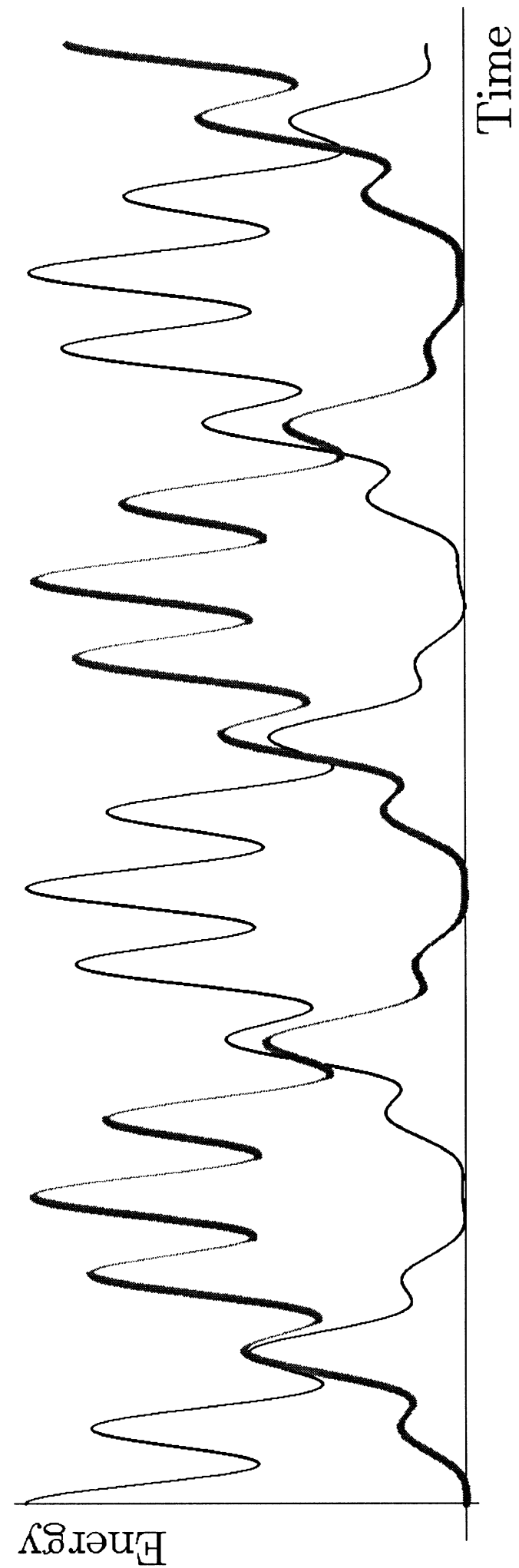

$\frac{\dot{0}}{\dot{D}}$ 


$$
\mathbb{N}
$$




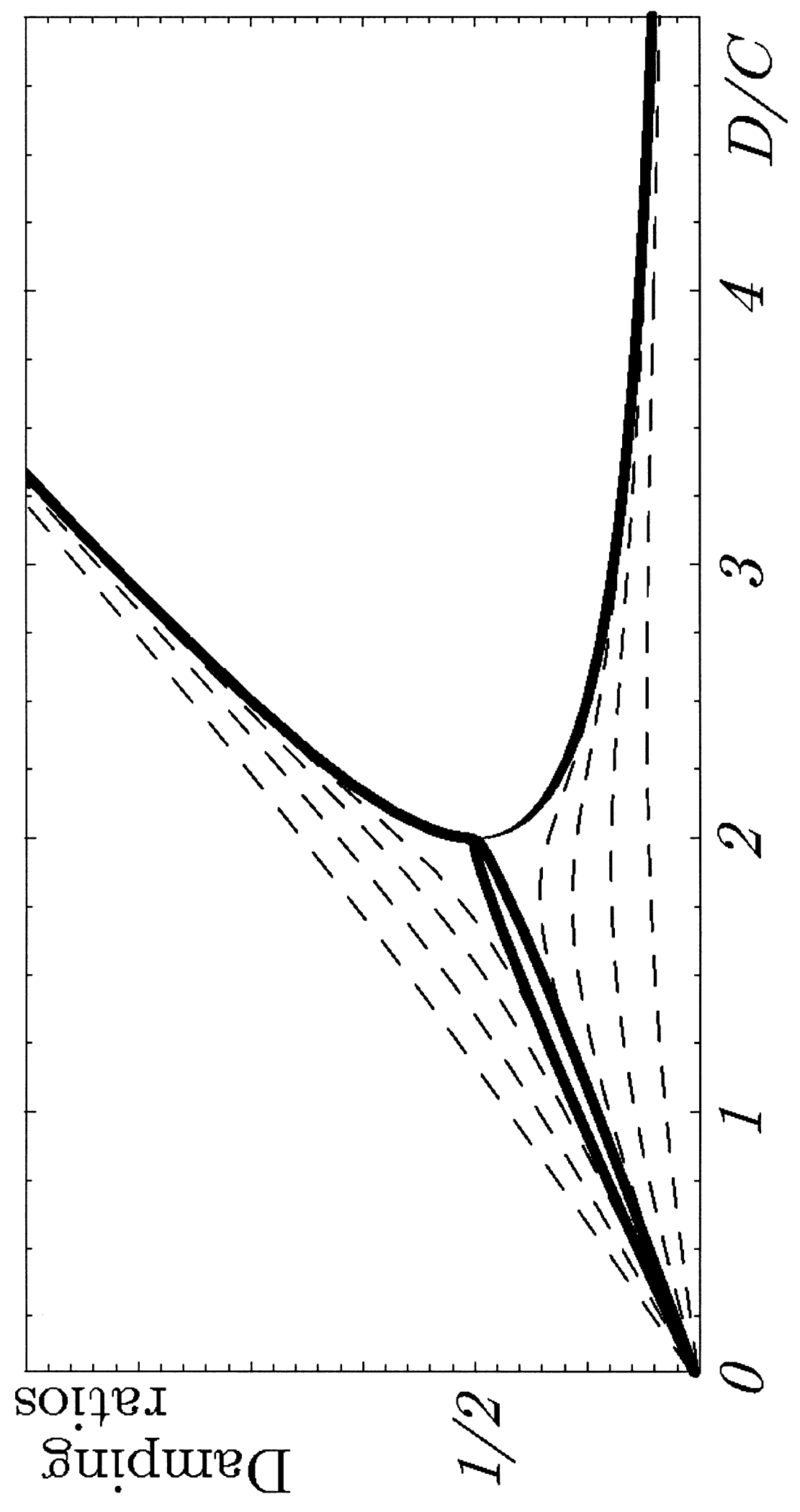

is 


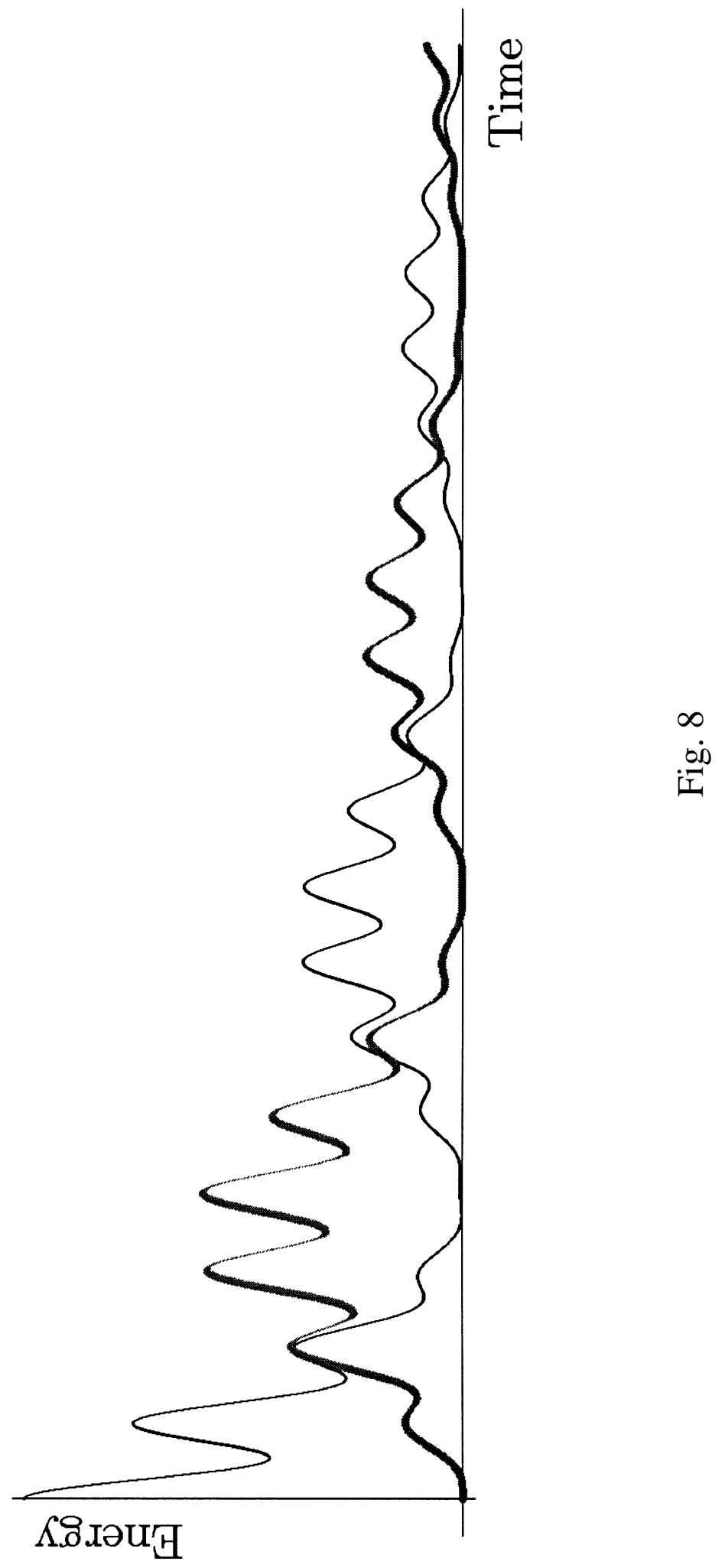





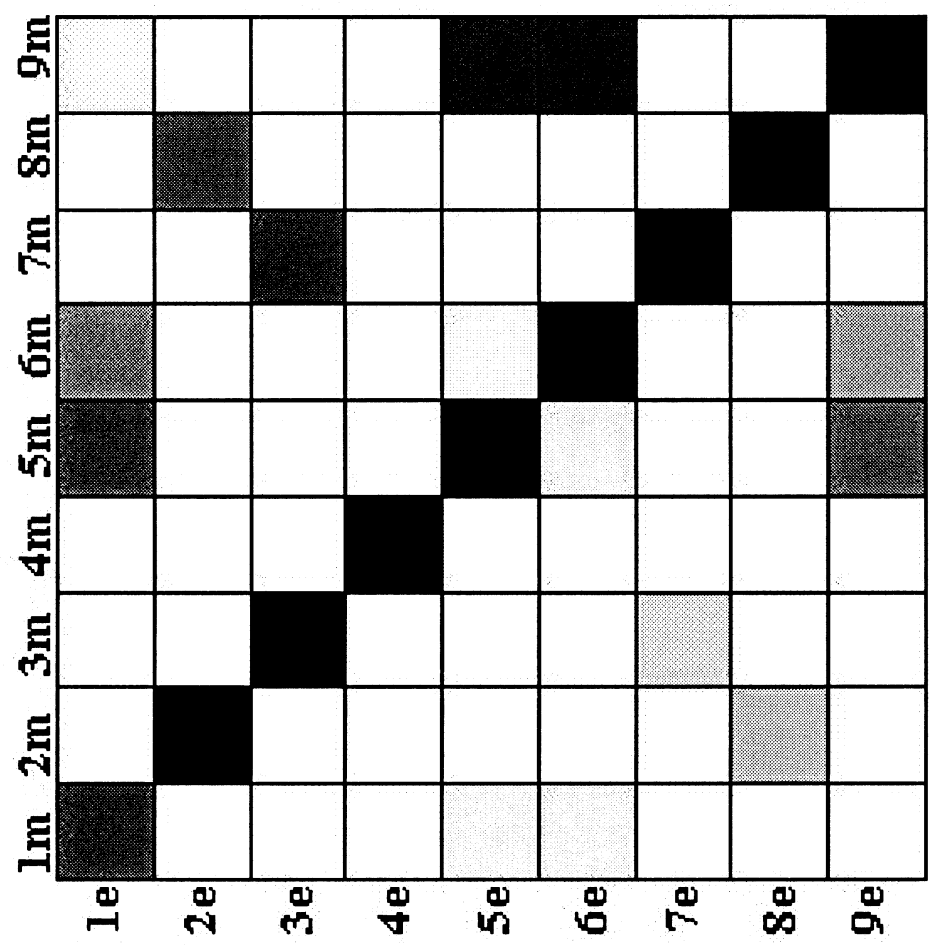

$\frac{0}{\dot{0}}$ 


$$
H
$$




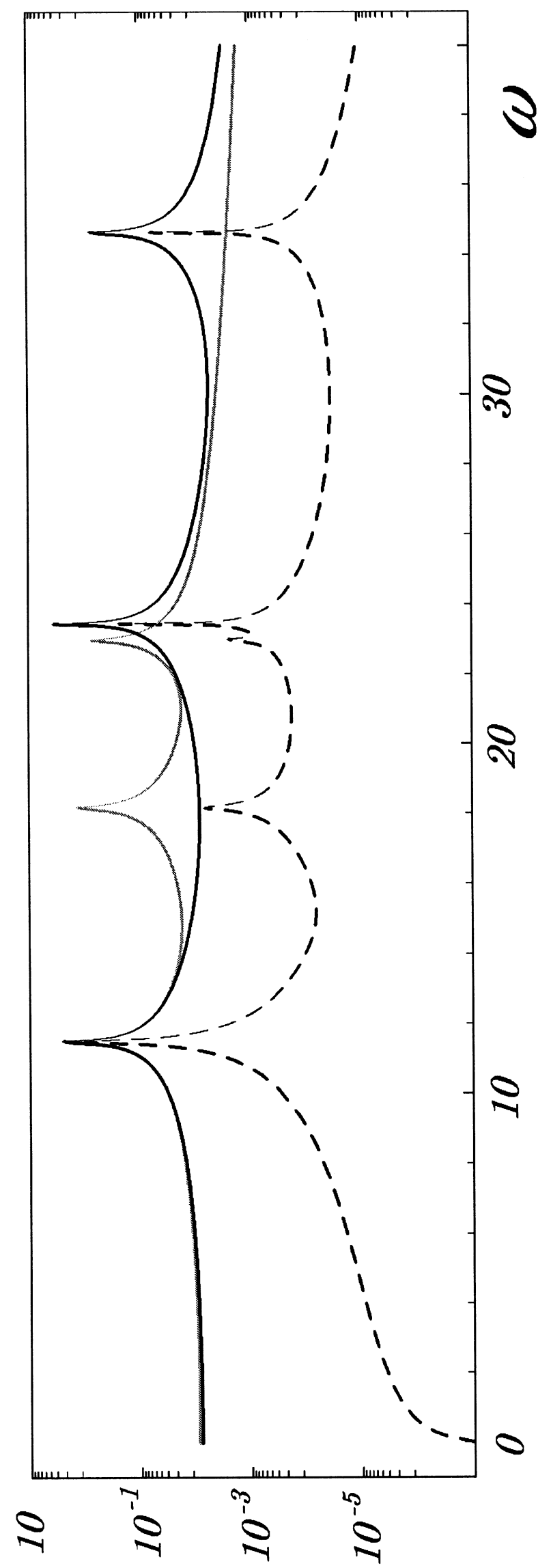

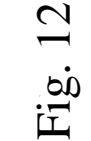




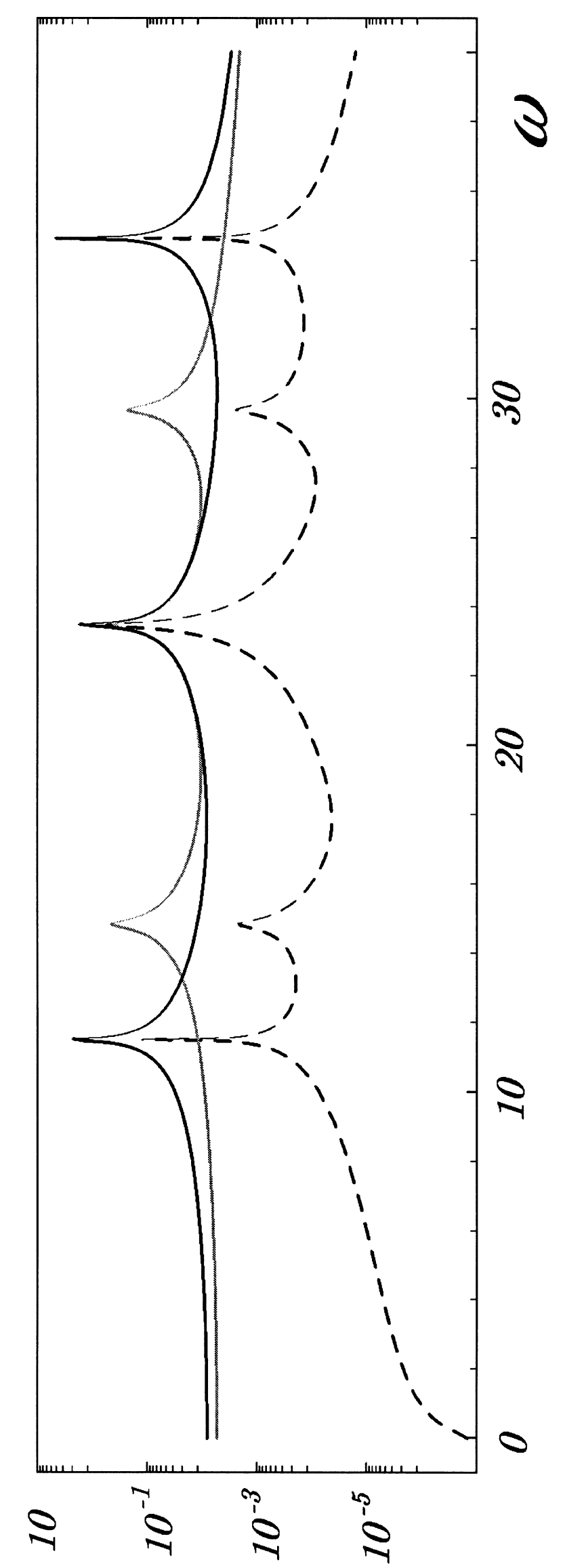

